\title{
Triple Therapy with Prednisolone, Pegylated Interferon and Sodium Valproate Improves Clinical Outcome and Reduces Human T-Cell Leukemia Virus Type 1 (HTLV-1) Proviral Load, Tax and $H B Z$ mRNA Expression in Patients with HTLV-1-Associated Myelopathy/Tropical Spastic Paraparesis
}

\author{
Reza Boostani $^{1} \cdot$ Rosita Vakili $^{2}$ - Samane Sadat Hosseiny ${ }^{3} \cdot$ Ali Shoeibi $^{1}$. \\ Bahare Fazeli $^{4} \cdot$ Mohammad Mehdi Etemadi $^{1} \cdot$ Faeze Sabet $^{5} \cdot$ Narges Valizade $^{4}$. \\ Seyed Abdolrahim Rezaee ${ }^{4}$
}

\begin{abstract}
Considering that there is no effective treatment for human T-cell leukemia virus type 1 (HTLV-1)-associated myelopathy/tropical spastic paraparesis, this study aimed to assess the impact of triple combination therapy-interferon- $\alpha$, valproic acid, and prednisolone - on clinical outcomes, main HTLV-1 viral factors, and host anti-HTLV-1 antibody response. HTLV-1 proviral load (PVL), and HBZ and Tax mRNA expression levels were measured in peripheral blood mononuclear cells of 13 patients with HTLV-1-associated myelopathy/tropical spastic paraparesis before and after treatment with $180 \mu \mathrm{g}$ pegylated interferon once a week, $10-20 \mathrm{mg} / \mathrm{kg} /$ day sodium valproate, and $5 \mathrm{mg} /$ day prednisolone for 25 weeks using a TaqMan real-time polymerase chain reaction assay. Furthermore,
\end{abstract}

Seyed Abdolrahim Rezaee

rezaeer@mums.ac.ir

1 Department of Neurology, Faculty of Medicine, Mashhad University of Medical Sciences, Mashhad, Iran

2 Center of Pathological and Medical Diagnostic Services, Iranian Academic Center for Education, Culture \& Research (ACECR), Mashhad Branch, Mashhad, Iran

3 Department of Biology, Science and Research Branch, Islamic Azad University, Kurdistan, Iran

4 Inflammation and Inflammatory Diseases Research Center, Faculty of Medicine, Mashhad University of Medical Sciences, Mashhad, Iran

5 Navid Medical Lab, Mashhad, Iran
anti-HTLV-1 titer, Osame Motor Disability Score, Ashworth spasticity scale, and urinary symptoms (through standard questionnaire and clinical monitoring) were assessed in patients before and after the treatment. HTLV-1 PVL and $H B Z$ expression significantly decreased after the treatment [PVL from $1443 \pm 282$ to $660 \pm 137$ copies $/ 10^{4}$ peripheral blood mononuclear cells ( $p=$ $0.01)$; and $H B Z$ from $8.0 \pm 1.5$ to $3.0 \pm 0.66(p<0.01)]$. Tax mRNA expression decreased after the treatment from $2.26 \pm$ 0.45 to $1.44 \pm 0.64$, but this reduction was not statistically significant $(p=0.10)$. Furthermore, anti-HTLV-1 titer reduced dramatically after the treatment, from $3123 \pm 395$ to $815 \pm 239(p<0.01)$. Clinical signs and symptoms, according to Osame Motor Disability Score and Ashworth score, improved significantly (both $p<0.01)$. Urinary symptoms and sensory disturbances with lower back pain were reduced, though not to a statistically significant degree. Although signs and symptoms of spasticity were improved, frequent urination and urinary incontinence were not significantly affected by the triple therapy. The results provide new insight into the complicated conditions underlying HTLV-1associated diseases.

Key Words HTLV-1 proviral load · HAM/TSP · Tax · HBZ · combination therapy $\cdot$ clinical symptoms

\section{Introduction}

Human T-cell leukemia virus type I (HTLV-1) is a retrovirus that has been extensively studied for $>30$ years [1]. Approximately 10-20 million people are infected with HTLV-1 
worldwide [2]. Endemic areas include South America, Southwestern Japan, Central Africa, the Melanesian Islands, the Caribbean basin, and the Middle East [3-5]. In Iran, HTLV1 is endemic at least in 2 provinces, including Khorasan Razavi and Northern Khorasan. However, HTLV-1associated disorders have been reported from other provinces such as Golestan, Alborz, and East Azarbayejan [3, 6-8]. About $2-5 \%$ of infected people develop adult T-cell leukemia/lymphoma (ATL) and HTLV-1-associated myelopathy/tropical spastic paraparesis (HAM/TSP) $[9,10]$. The virus may also be a risk factor for infective dermatitis, uveitis [10], and even cardiovascular diseases [11].

HAM/TSP, a neuroinflammatory disorder of the central nervous system, is associated with perivascular and parenchyma infiltration of HTLV-1-infected T cells and activated cytotoxic T lymphocytes (CTL) [12]. A previous study has shown a significantly higher proviral load (PVL) in patients with HAM/TSP compared with asymptomatic carriers, suggesting that active HTLV-1 viral replication plays a critical role in the development of the disease [13]. In addition, HTLV-1-infected human $\mathrm{CD}^{+}$T cells express viral regulatory proteins such as Tax and HTLV-1 bZIP factor (HBZ) to provide virus survival and dissemination. Tax, which is a transcription activator in the early stages of infection, induces Tcell proliferation and subsequently cellular transformation by modulation of cellular transcription factors [9]. Moreover, Tax is the immunodominant target antigen recognized by the CTL response [14]. This protein contributes to the development and progression of HTLV-1-associated diseases [15]. HBZ is a regulatory gene in the later stages of the infection, and suppresses the expression of Tax to escape from the specific CTLs [16]. HBZ expression has a positive effect on HTLV-1 viral replication [17]. Moreover, a significant positive correlation has been previously reported between HBZ mRNA expression and HAM/TSP disease severity [18-20]. HTLV-1infected cells circulate in the body; hence, blood samples provide an easily accessible source of information and, in particular, a large number of viruses for the understanding and monitoring of HTLV-1-associated diseases. Immune system disruption mechanisms in patients with HAM/TSP have been extensively investigated $[9,21]$. The immune suppression and deregulation of the immune system in HTLV-1-infected people result in autoimmunity to neurons and provide a microenvironment for inducing HAM/TSP. This immunosuppressive microenvironment enables HTLV-1-infected cells to evade from the host immune responses and induce HTLV-1associated diseases [20].

Studies on HTLV-1 as an agent involved in deregulation of the immune system have shown controversial results, and after years of research on HTLV-1 and associated diseases, treatment of HAM/TSP still remains a challenge for clinicians [22]. Treatments for patients with HAM/TSP are divided into symptomatic and etiologic groups. Symptomatic treatments include antispastic and anticholinergic agents, analgesics, physiotherapy, and the management of emotional and social problems [10]. In etiological treatments, corticosteroids, cytotoxic agents, interferon (IFN)- $\alpha$, plasma exchange, and other immunomodulatory agents such as danazol, erythromycin, phosphomycin, sulfasalazine, and pentoxifylline can be used. It should be considered that, owing to the slow progression of the disease, the chance of improvement is increased during the first [10, 23]. Although glucocorticoid therapy has been relatively effective in improving motor function, patients who fail to respond to corticosteroids or those who cannot tolerate it because of adverse effects, plasma exchange or IFN- $\alpha$ could be used [10, 24-27]. Recently, IFN- $\alpha$ was used in patients with HAM/TSP, and clinical and immunological improvements were assessed. Six-month treatment had a temporary positive effect on motor disability, spasticity, and urinary disturbances [25]. Moreover, a remarkable decrease in HTLV-1 viral load, antibody level, and lymphocyte and monocyte counts was observed. However, adverse effects such as fever, chills, weakness, malaise, alopecia, and depression were observed in $64 \%$ of the patients. The partial improvement observed with IFN- $\alpha$ therapy suggests that viral factors and immune modulatory factors play important roles in the pathophysiology of HAM/TSP. Consequently, antiviral drugs combined with the immunomodulators may be more effective for the treatment of patients with HAM/TSP. Our recent study [20] demonstrated that triple therapy with arsenic, IFN- $\alpha$, and zidovudine shifts the cytokine expression from a regulatory T cell and T helper 2 phenotype to a T helper 1 phenotype. This shift enhances the eradication of ATL cells and prevents the emergence of opportunistic infections. Therefore, the present study assessed the effect of a novel combination therapy with 3 agents: IFN- $\alpha$, as an immunomodulator; valproic acid as an agent that promotes histone hyperacetylation and HTLV15 '-promoter-driven transcription activator; and prednisolone as an anti-inflammatory agent on clinical outcomes and main HTLV-1 viral factors, such as Tax, HBZ, and PVL, as well as anti-HTLV-1 titer, which is indicative of the host immune response in patients with HAM/TSP. In addition to the clinical improvement, the current study provides new insights into the effect of this combination on viral behavior and host defense.

\section{Methods}

\section{Study Design}

A cross-sectional study was carried out from February 2011 to November 2012 on 13 patients with HAM/TSP in the HTLV1 Foundation Clinic of Ghaem Hospital, Mashhad University of Medical Sciences (MUMS), Mashhad, Iran. The Biomedical Research Ethics Committee of Mashhad University of Medical Sciences approved only 13 patients to be treated in 
the study (Number: 900637). After receiving formal informed consent, a standardized clinical checklist, including demographic information and the clinical current illness, were completed by a trained neurologist. HTLV-1 infection was determined using a HTLV-1 antibody serological test and confirmed by polymerase chain reaction (PCR) for Tax and long terminal repeat regions as previously described [3]. The inclusion criteria were the presence of HTLV-1 antibodies and DNA in serum; confirmation of intrathecal anti-HTLV-1 antibody synthesis and/or the presence of HTLV-1 DNA in the cerebrospinal fluid; age of 18-55 years; and a diagnosis of HAM/TSP according to modified diagnostic guidelines for HAM/TSP (used since 1987) [28]. Moreover, the patients had no history of previous treatment for HAM/TSP, or concomitant treatment. Patients with heart failure, chronic renal failure, liver and thyroid disorders, HIV, hepatitis B virus infections, thrombocytopenia, anemia, or leukopenia, or pregnant or nursing women, and patients with a known hypersensitivity to IFN, were excluded from the study.

\section{Clinical Evaluation}

The Osame Motor Disability Score (OMDS), which ranges from 0 (normal walking and running) to 13 (completely bedridden) [28], and the Ashworth spasticity scale (ASS) [29] were measured before and after the treatment. In addition, urinary disturbances based on the Incontinence Questionnaire-Urinary Incontinence Short Form and clinical examination such as urinary frequency, incontinence, and feeling of incomplete emptying were evaluated before and after the treatment [30-32]. A positive response was mainly considered as an improvement in motor function, urinary symptoms, and neurologic signs.

\section{Treatment Protocol}

Patients were treated with $180 \mu \mathrm{g}$ pegylated IFN (peg-IFN) once a week (Roche, Mannheim, Germany), 10-20 mg/kg/ day valproate sodium (Sandoz, Holzkirchen, Germany) and $5 \mathrm{mg} /$ day prednisolone (Aburaihan, Tehran, Iran) for up to 25 weeks. Complete blood count and liver function tests were carried out to investigate possible complications during this period as follows: weekly during the first month; every 2 weeks during the second and third months; and monthly thereafter in the absence of any abnormal results. Thyroid function tests were performed during the first, third, and sixth months. Urinary symptoms, OMDS and ASS score were evaluated every 3 months. After 8 weeks of treatment, patients who demonstrated no clinical improvement were excluded from the study. In those who had a clinical improvement, the treatment was continued over 25 weeks following a consultation with a neurologist. However, follow-up of the patients after stopping triple therapy was not included in this study.

\section{Laboratory Methods}

\section{Measurement of HTLV-1 PVL}

Blood samples were collected from patients prior to and after 6 months of treatment. Cellular DNA was extracted from peripheral blood mononuclear cells (PBMC) using a commercial kit (QIAamp DNA Blood Mini Kit; Qiagen, Hilden, Germany). PBMC were isolated from ethylenediaminetetraacetic acid-treated blood samples by Ficoll density gradient (Sigma, Schnelldorf, Germany). A real-time PCR assay was performed by a Rotorgen Q Real-Time PCR machine (Qiagen) using a commercial absolute quantification kit (Novin Gene, Tehran, Iran). To measure HTLV-1 PVL in PBMC, specific primers and a fluorogenic probe were used. The HTLV-1 copy number was reported as an actual amount of cellular DNA by means of albumin gene quantification as the reference gene. Then, HTLV-1 and albumin DNA concentrations from two 5point standard curves were calculated. The normalized HTLVI PVL values were measured as the ratio of (HTLV-1 DNA copies number/albumin DNA copies number/2) $\times 10^{4}$ and expressed as the number of HTLV-1 proviruses per $10^{4} \mathrm{PBMC}$ [25].

\section{HBZ and Tax mRNA Expression}

Cellular RNA was extracted from PBMC using TriPure Isolation Reagent (Roche Diagnostics, Lewes, UK). Complementary DNA was then synthesized using TaqMan Gold RT-PCR Kit (Takara, Otsu, Shiga, Japan), according to the manufacturer's instructions. A quantitative real-time PCR was performed for HTLV-1 HBZ and Tax mRNA, and a cellular reference gene, $B 2 M$ [33], using TaqMan method reagent target kits (Applied Biosystems, Foster City, CA, USA) in accordance with the manufacturer's instructions. Primers and probes for Tax, $H B Z$, and $B 2 M$ mRNA expression are summarized in Table 1. PCR conditions were as follows: holding at $94{ }^{\circ} \mathrm{C}$ for $5 \mathrm{~min}$, followed by 45 cycles of denaturation at $94{ }^{\circ} \mathrm{C}$ for $30 \mathrm{~s}$, annealing at $55^{\circ} \mathrm{C}$ for $30 \mathrm{~s}$ and elongation at $72{ }^{\circ} \mathrm{C}$ for $30 \mathrm{~s}$, and final extension at $72{ }^{\circ} \mathrm{C}$ for $5 \mathrm{~min}$. Realtime PCR was performed in a Rotor Gene Q 6000 (Qiagen,) and data were analyzed with the 2 standard curves relative method. Standard curves were prepared for target and reference genes using the TaqMan method with Rotor Gene 6000 software (Qiagen), and the data were analyzed. The relative quantity of each mRNA was normalized to the relative quantity of $B 2 M$ mRNA.

\section{HTLV-1 Antibody Titration}

Specific HTLV-1 antibody titration was carried out before and after the treatment, as previously described [25]. Sera were collected and frozen at $-40^{\circ} \mathrm{C}$. An endpoint ELISA dilution 
Table 1 Primer and probe sequences used in quantitative real-time polymerase chain reaction

\begin{tabular}{llll}
\hline Targeted gene & Sequence (5' to 3') & Purpose & Product size (base pairs) \\
\hline Tax & ATCCC GTGG AGACTCCTCAA & Forward & \multirow{2}{*}{100} \\
& CCTGGGAAGTGGGCCATG & Reverse & \\
& CATGCCCAAGACCCGTCGGAGG & Probe & \\
HBZ & CTCGACCTGAGCTTTAAACTTACC & Forward & 133 \\
& CATGACACAG GCAAGCATCG & Reverse & \\
& CGGACGCAGTTCAGGAGGCACCAC & Probe & \\
\hline
\end{tabular}

method (Diapro, Milan, Italy) was carried out to assess HTLV1 antibody titer by 2 -fold dilution.

\section{Statistical Analysis}

The collected data were initially entered into SPSS version 13 (IBM, Armonk, NY USA). Normality of the data was checked prior to the data analysis. Clinical impact, Tax mRNA expression and HTLV-1 PVL before and after the treatment were compared using the paired sample $t$ test. The Wilcoxon matched paired $t$ test was used to compare $H B Z$ mRNA expression and anti-HTLV-1 titer before and after the treatment. Descriptive data were summarized as means and SEM. $p$ values were considered statistically significant if they were $\leq$ 0.05 .

\section{Results}

\section{Demographic Data and Clinical Impact}

In this study, 13 patients [mean age $40.2 \pm 3.7$ years (range 20-55 years)] with HAM/TSP were enrolled (9 women, 4 men). Mean OMDS improved significantly, as demonstrated by a decrease from $2.4 \pm 1.9$ before treatment to $1.3 \pm 1.8$ after treatment $(p<0.01)$. The mean ASS score also improved significantly, decreasing from $2.0 \pm 1.1$ to $0.9 \pm 1.2$ $(p<0.01)$. However, the reduction in urinary symptoms was not significant. Moreover, the difference in sensory disturbances with low back pain, before and after the treatment, did not achieve significance at the $95 \%$ confidence interval $(p=0.06)$. Tables 2 and 3 show the clinical conditions of the patients before and after the 6 months of triple therapy.

\section{Laboratory Findings}

\section{Anti-HTLV-1 Antibody Titer and HTLV-1 PVL}

The mean anti-HTLV-1 antibody titer decreased significantly from $3123 \pm 395$ before treatment to $815 \pm 239$ after treatment ( $p<0.01$; Fig. 1). In addition, the mean HTLV-1 PVL before treatment was $1443 \pm 282$ copies $/ 10^{4} \mathrm{PBMC}$ (range 293-3802 copies $/ 10^{4}$ PBMC), and the mean HTLV-1 PVL after treatment was $660 \pm 137$ copies $/ 10^{4}$ PBMC (range 62-1822 copies $\left./ 10^{4} \mathrm{PBMC}\right)$, indicating a significant reduction $(p=0.01$; Fig. 2). Furthermore, there was a statistically significant correlation between HTLV-1 PVL and the age of the patients ( $r=$ $0.59 ; p=0.01)$. Inconsistent findings have been reported in the literature regarding the correlation between PVL and age [34, 35]. Although Furtado et al. [34] did not find any correlation between PVL and age at blood sampling, age at disease onset, or duration of disease, Matsuzaki et al. [35] reported a higher PVL in patients with a disease onset at an age of $>65$ years compared with those with a younger age of onset [35].

\section{HTLVN1 Tax and HBZ Expression}

Mean Tax mRNA expression in patients with HAM/TSP tended to decrease from 2.26 (range 2.83-1.71) before the treatment to 1.44 (range 2.24-0.90) after the treatment but failed to achieve statistical significance (Fig. 3). Notably, the mean $H B Z$ mRNA expression decreased by two-thirds from
Table 2 Clinical conditions of patients with human T-cell leukemia virus type 1 -associated myelopathy/tropical spastic paraparesis before and 6 months after triple therapy in the group of 13 patients (b) for individual patients

\begin{tabular}{llll}
\hline Clinical impact & Before treatment & After treatment & $p$-value \\
\hline OMDS & $2.4 \pm 1.9$ & $1.3 \pm 1.8$ & $<0.01$ \\
ASS & $2.0 \pm 1.1$ & $0.9 \pm 1.2$ & $<0.01$ \\
Frequent urination & $1.1 \pm 0.8$ & $0.8 \pm 0.7$ & 0.20 \\
Urinary incontinence & $0.9 \pm 0.8$ & $0.8 \pm 1.1$ & 0.50 \\
Feeling of incomplete voiding & $0.9 \pm 0.7$ & $0.6 \pm 0.8$ & 0.08 \\
Sensory disturbances with low back pain & $3.1 \pm 3.3$ & $1.4 \pm 1.9$ & 0.06 \\
\hline
\end{tabular}

Data are shown as mean \pm SEM, and analyzed using the paired sample $t$ test

OMDS $=$ Osame Motor Disability Score; ASS=Ashworth spasticity scale 
Table 3 Clinical conditions of patients with human T-cell leukemia virus type 1-associated myelopathy/tropical spastic paraparesis before and 6 months after triple therapy individual patients

\begin{tabular}{|c|c|c|c|c|c|c|c|c|c|c|c|c|c|}
\hline \multirow{2}{*}{$\begin{array}{l}\text { Patient } \\
\text { number }\end{array}$} & \multirow[t]{2}{*}{ Sex } & \multirow{2}{*}{$\begin{array}{l}\text { Age } \\
\text { (years) }\end{array}$} & \multirow{2}{*}{$\begin{array}{l}\text { Duration of } \\
\text { disease (years) }\end{array}$} & \multicolumn{2}{|l|}{ OMDS } & \multicolumn{2}{|l|}{ ASS } & \multicolumn{2}{|c|}{ Frequent urination } & \multicolumn{2}{|c|}{ Anti-HTLV-1 titer } & \multicolumn{2}{|c|}{ HTVL-1 proviral load } \\
\hline & & & & Before & After & Before & After & Before & After & Before & After & Before & After \\
\hline 1 & $\mathrm{~F}$ & 51 & 4.0 & 4 & 2 & 2 & 1 & 2 & 1 & 3200 & 800 & 1520 & 732 \\
\hline 2 & M & 54 & 1.5 & 1 & 0 & 1 & 0 & 0 & 0 & 4800 & 400 & 769 & 378 \\
\hline 3 & $\mathrm{~F}$ & 27 & 1.0 & 3 & 0 & 2 & 0 & 0 & 0 & 1600 & 200 & 520 & 62 \\
\hline 4 & M & 54 & 16.0 & 1 & 0 & 1 & 0 & 1 & 1 & 3200 & 200 & 293 & 925 \\
\hline 5 & $\mathrm{~F}$ & 40 & 2.0 & 1 & 0 & 1 & 0 & 2 & 0 & 4800 & 400 & 2864 & 144 \\
\hline 6 & $\mathrm{~F}$ & 55 & 6.0 & 1 & 0 & 1 & 0 & 0 & 1 & 1400 & 400 & 751 & 284 \\
\hline 7 & $\mathrm{~F}$ & 32 & 8.0 & 6 & 4 & 3 & 2 & 2 & 2 & 4800 & 3200 & 1202 & 732 \\
\hline 8 & M & 55 & 20.0 & 3 & 1 & 2 & 1 & 2 & 2 & 3200 & 800 & 963 & 402 \\
\hline 9 & $\mathrm{~F}$ & 31 & 13.0 & 5 & 5 & 2 & 2 & 2 & 2 & 1600 & 1600 & 3802 & 1295 \\
\hline 10 & $\mathrm{~F}$ & 51 & 2.0 & 1 & 1 & 1 & 1 & 1 & 0 & 800 & 400 & 638 & 429 \\
\hline 11 & $\mathrm{~F}$ & 27 & 2.0 & 4 & 2 & 2 & 1 & 2 & 1 & 3200 & 400 & 2166 & 1822 \\
\hline 12 & M & 20 & 6.0 & 1 & 0 & 1 & 0 & 1 & 1 & 4800 & 200 & 1893 & 969 \\
\hline 13 & $\mathrm{~F}$ & 26 & 3.0 & 1 & 0 & 1 & 0 & 1 & 1 & 3200 & 1600 & 1376 & 408 \\
\hline
\end{tabular}

$\mathrm{M}=$ male; $\mathrm{F}=$ female; $\mathrm{OMDS}=$ Osame Motor Disability Score; ASS=Ashworth spasticity scale score

$8.0 \pm 1.5$ (range 9.91-6.73) before triple therapy to $3.0 \pm 0.66$ (range 3.31-2.13) after treatment ( $p<0.01$; Fig. 4). These results demonstrate that the main impact of this triple therapy is on $H B Z$ activity, which decreases the ability of the virus to evade the host immune response.

\section{Discussion}

HAM/TSP is a chronic inflammatory disease caused by HTLV-1 infection in the spinal cord. Although most HTLV-

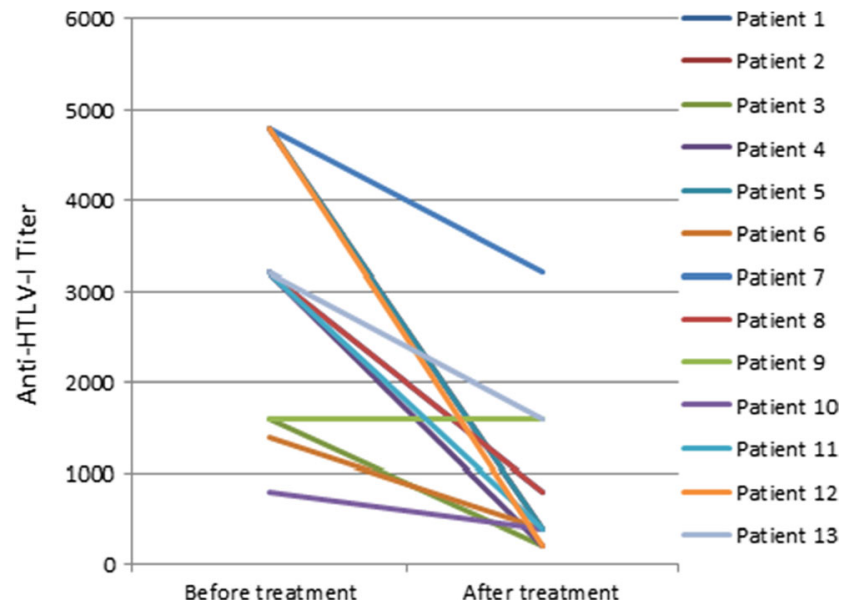

Fig. 1 Comparison of anti-human T-cell leukemia virus type 1 (HTLV-1) titer before and after treatment in patients with HTLV-1-associated myelopathy/tropical spastic paraparesis. Anti-HTLV-1 antibody titer decreased significantly after the treatment (Wilcoxon matched paired $t$ test $p<0.01)$. An endpoint dilution ELISA was carried out to assess HTLV-1 antibody titer by 2 -fold dilution 1-infected individuals remain asymptomatic carriers, approximately $5 \%$ of infected individuals develop HAM/TSP, which is associated with lower extremity weakness, spasticity, urinary disturbance, and sensory symptoms. Despite numerous studies of HAM/TSP, its pathophysiology remains poorly understood [36]. Therefore, an effective treatment is still elusive. In 1990, 5 patients with HAM/TSP were treated for the first time with IFN- $\alpha, 4$ of whom showed improvement of gait and

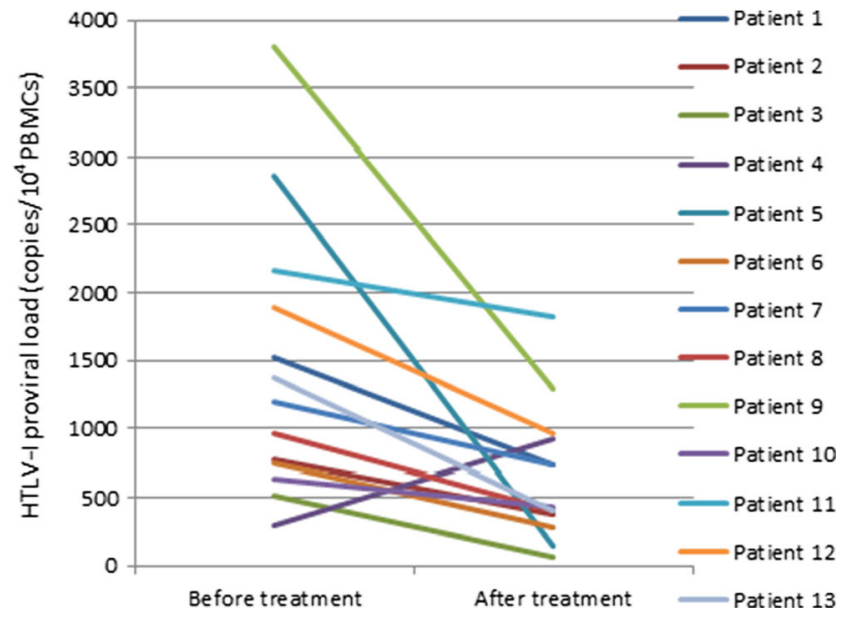

Fig. 2 Human T-cell leukemia virus type 1 (HTLV-1) proviral load in patients with HTLV-1-associated myelopathy/tropical spastic paraparesis before and after the treatment. Mean HTLV-1 proviral load decreased significantly after the treatment (paired sample $t$ test $p=0.01$ ). Two standard curves of absolute real-time polymerase chain reaction for Tax and albumin copy numbers were carried out on peripheral blood mononuclear cells (PBMC) and the HTLV-1 proviral load assessed using the equation (HTLV-1 DNA copy number/albumin DNA copy number $/ 2) \times 10^{4}$ and expressed as the number of HTLV-1 proviruses per $10^{4} \mathrm{PBMC}$ 


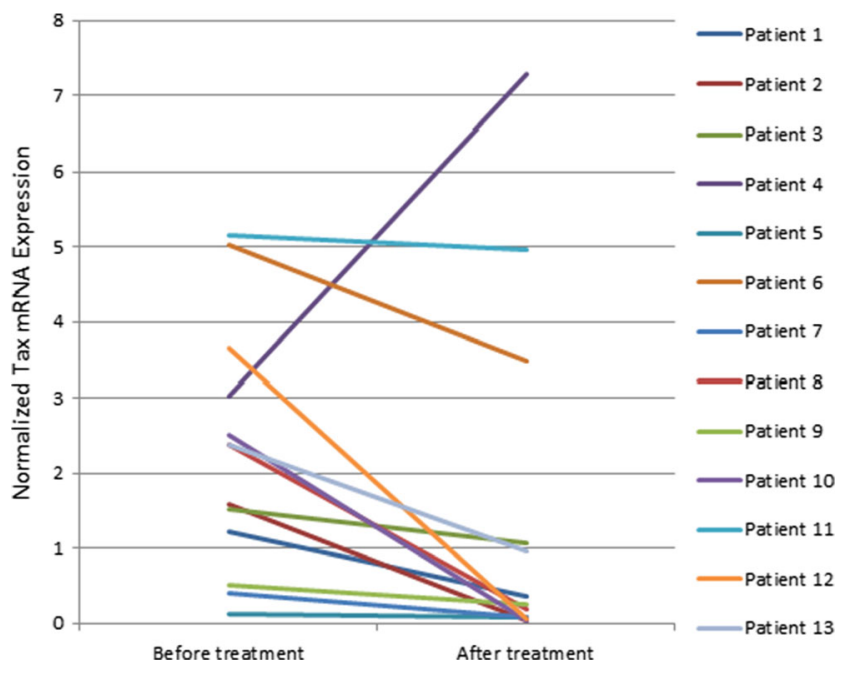

Fig. 3 Tax mRNA expression before and after the treatment in patients with human T-cell leukemia virus type 1-associated myelopathy/tropical spastic paraparesis. Mean Tax mRNA expression decreased but did not achieve significance (paired sample $t$ test $p=0.10$ ). Tax and B2M mRNA expression were assessed by 2 standard curves relative to the TaqMan real-time polymerase chain reaction method, and the relative quantity of Tax mRNA was normalized to the relative quantity of $B 2 M$ mRNA

sensory and/or sphincter disturbance [37]. In other studies, at least 4 weeks of treatment with IFN- $\alpha$ showed a relative improvement (33-88 \%) of clinical symptoms, particularly motor disability, but no significant improvement of urinary disturbance was observed $[38,39]$. Our previous study of IFN- $\alpha$ treated patients with HAM/TSP showed a significant but temporary effect in motor and bladder functions [25]. PVL significantly decreased in responders and nonresponders, 1 and 6 months after the treatment. Flow cytometry showed no

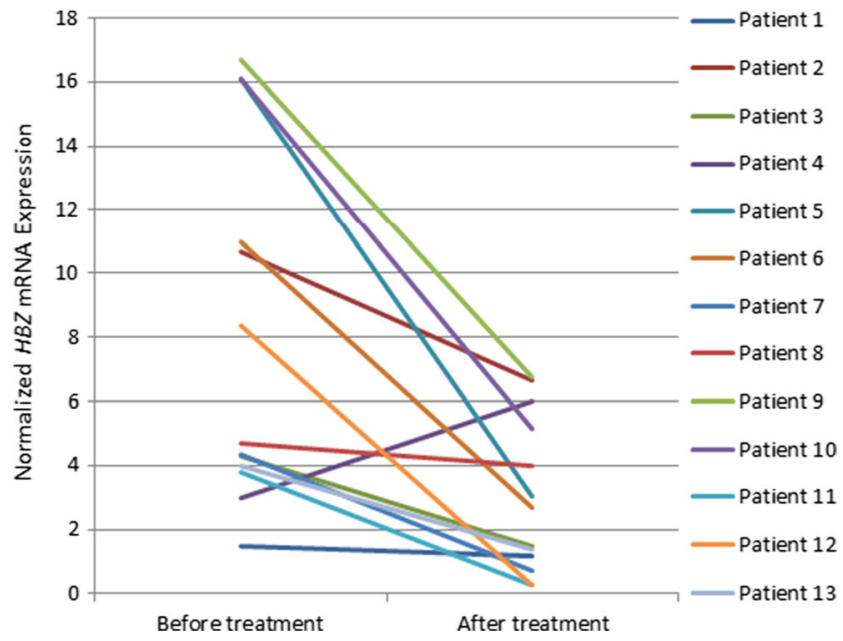

Fig. $4 \mathrm{HBZ}$ mRNA expression before and after the treatment in patients with human T-cell leukemia virus type 1-associated myelopathy/tropical spastic paraparesis. Mean $H B Z$ expression decreased significantly after the treatment (Wilcoxon matched paired $t$ test $p<0.01$ ). $H B Z$ and B2M mRNA expression were assessed by 2 standard curves relative to TaqMan real-time polymerase chain reaction, and the relative quantity of $\mathrm{HBZ}$ mRNA was normalized to the relative quantity ofB $\beta 2 \mathrm{M}$ mRNA significant changes in $\mathrm{CD} 4, \mathrm{CD} 8, \mathrm{CD} 4 \mathrm{CD} 25$ and $\mathrm{CD} 16$ CD56 counts [25]. This study revealed the positive effect in patients with HAM/TSP of IFN- $\alpha$ during the treatment period [25]. Therefore, therapeutic protocols were designed to interfere with the HTLV-1-infected cell proliferation or virus replication [19]. The principle aim was to activate viral gene expression and thereby expose virus positive cells to the host immune response; however, this approach was still ineffective. Recently, a great deal of attention has been focused on sodium valproate, which is well tolerated and displays adequate pharmacokinetics. This compound inhibits histone deacetylation and activates HTLV-1 5'-promoter-driven transcription [40]. However, some concerns have been raised about this therapeutic application, as the $\mathrm{CD} 8^{+}$-cell antiviral function might be altered after the treatment [19]. Moreover, memory $\mathrm{CD}^{+} \mathrm{T}$ cells might be destroyed by autologous HTLV-1-specific CTL [19]. A recent study indicated that sodium valproate, as a histone deacetylase inhibitor, has positive effects on the reduction of PVL and better recognition of infected cells by the immune system. In this study, combination therapy of valproate and azidothymidine in infected asymptotic monkeys revealed an increase in the cytotoxic T-cell population and a significant decline in PVL [41]. Nevertheless, in the study conducted in 19 patients with HAM/TSP, treatment with $20 \mathrm{mg} / \mathrm{kg} /$ day oral valproate for 24 months did not show a remarkable effect on HTLV-1 PVL, efficiency of $\mathrm{CD}^{+}$lysis, or HAM/TSP disability. However, walking time in 3 patients improved significantly [42].

Therefore, 20 years of investigation to find an effective treatment for HAM/TSP and ATL led us to test a combination therapy of peg-IFN as an immunomodulator, sodium valproate as a deacetylase inhibitor, and prednisolone as an anti-inflammatory agent in patients with HAM/TSP [10, 20, $25,43]$. In the present study, significant improvements were observed in clinical aspects such as motor disability, spasticity, and antibody titer of HTLV-1 virus after 6 months of triple therapy; however, urinary findings and sensory disturbances with lower back pain improved marginally. It has previously been reported that HTLV-1 PVL decreases after treatment with IFN- $\alpha$ in patients with HAM/TSP $[39,44]$. The results of our study with triple treatment also showed a significant reduction in HTLV-1 PVL. The reduction in HTLV-1 PVL might be due to the direct antiviral effect of IFN- $\alpha$ and its ability to potentiate major histocompatibility complex class I expression and, as a consequence, enhance the killing ability of infected cells by cytolytic T lymphocytes $\left(\mathrm{CD}^{+}\right)$. Lezin et al. [40] demonstrated that valproate can reduce HTLV-1 PVL; therefore, the combination of IFN- $\alpha$ and valproate in these patients resulted in a significant decline in HTLV-1 PVL. Furthermore, downregulation of HBZ expression by triple therapy in the current study may prevent the evasion mechanism of the virus and, consequently, may render infected cells more vulnerable to immune responses. 
Elevated HTLV-1 PVL in patients with HAM/TSP reportedly correlates with higher $H B Z$ mRNA expression and disease severity [19]. HBZ is a potent suppressor of Tax-mediated virus gene transcription and plays a critical role in the tightly regulated pattern of HTLV-1 gene expression [19]. As discussed before, the principle of using sodium valproate was to activate HTLV-1 gene expression, thereby exposing virus-positive cells to the host immune response in order to eliminate the infected cells. Belrose et al. [19] demonstrated that valproate can induce such activity and bring the HTLV-1infected cells to the effector mechanism of the immune system through temporary activation of virus expression, blocks HBZ expression, and counterbalances Tax stimulation of virus replication.

Some related studies suggest that sodium valproate treatment of patients with HAM/TSP increases peripheral blood PVL during the first week of the treatment [19]. Addition of peg-IFN as an immunomodulator to valproate, blocks virus propagation, and prevents the transient elevation of virus replication [19]. In addition to Tax expression and the Tax-mediated CTL response, another mechanism involving $H B Z$ repression might affect the outcome of sodium valproate therapy [19]. Furthermore, in the present study, after 6 months of treatment, Tax expression decreased; however, the result was not statistically significant. It seems that, $H B Z$ expression reduces after triple therapy owing to immunomodulation of IFN- $\alpha$ and the epigenetic effects of valproate. Therefore, enforced anti-HTLV-1-specific CTLs could recognize HTLV-1-infected cells. Moreover, prednisolone suppresses the specific CTLs simultaneously. As a result, HTLV-1 PVL and disease severity decrease after this combination therapy. According to the treatment protocol of IFN- $\alpha$ in hepatitis B and $\mathrm{C}$ virus infections, it seems that the longer treatment might have a better impact on clinical improvement; however, there is not enough evidence for this in HTLV-1 infection. Lezin et al. [40] showed that valproate treatment for 2 years was safe in patients with HAM/TSP. As the virus is integrated into the host genome, eradication of the virus appears to be impossible. Therefore, there was no rational reason to continue triple therapy for $>$ 6 months, Moreover, the present study has its own limitations. First, the number of patients with HAM/TSP and the length of therapeutic intervention approved by the ethics committee; and, second, funding limitations made the treatment unaffordable for the researchers and patients.

Valproate has been used clinically for a long time in many neuropsychiatric disorders, and recently for HIV and cancers, in which it is used as a sensitizing agent within a polytherapy regimen $[45,46]$. However, valproate is a known human teratogen when administered during the first trimester of pregnancy [47, 48]. Valproate acts on class I and II histone deacetylases, which collectively deacetylate a variety of nuclear and cytoplasmic proteins, so its effects are inevitably pleiotropic. For example, valproate induces oxidative stress, with increased levels of reactive oxygen species; thus, antioxidants can improve some of its side effects $[18,19]$. Our previous studies (Boostani et al., unpublished data), and a recent Japanese study [49], showed that the prescription of antioxidant agents had positive or improving effects on signs and symptoms of HAM/TSP. Therefore, it could be suggested that the addition of a well-tolerated antioxidant in combination with valproate might have a better impact on HTLV-1-associated diseases.

In summary, combination therapy of peg-IFN as an immunomodulator, sodium valproate as a deacetylase inhibitor, and prednisolone as an anti-inflammatory agent demonstrated a remarkable decrease in HTLV-1 PVL, anti-HTLV-1 antibody titer, HBZ and Tax expression, and a significant improvement in motor disability and spasticity in patients with HAM/TSP after 6 months of treatment. Although there was a remarkable improvement in spasticity, we observed no significant difference in urinary problems with the triple therapy. Besides Tax expression and the Taxmediated CTL response, $H B Z$ also plays important roles in HAM/TSP manifestation. Moreover, repression of $H B Z$ might be the effect of sodium valproate therapy. Therefore, in this triple therapy, sodium valproate might repress $H B Z$ expression and counterbalance Tax stimulation of virus replication and T-cell proliferation. In addition to upregulation of virus activity (valproate), suppression of inflammatory reactions (prednisolone) and modulation of antiviral immune-responses (IFN- $\alpha$ ), other mechanisms involved in HAM/TSP manifestation might be considered for the treatment of HTLV-1associated inflammatory diseases. Furthermore, no significant adverse effects were documented during 25 weeks of combination therapy. These findings may provide a new insight into the complex immune conditions underlying HTLV-1-associated diseases and suggest personalized treatment based on viral and host immune markers. Therefore, further studies with larger sample sizes and some modifications, such as the addition of an antioxidant, are necessary to improve the strategy of HAM/TSP treatment.

Acknowledgments We thank Navid Medical Laboratory, Mashhad, Iran, for performing the tests. This work was supported by Vice Chancellor for Research, Mashhad University of Medical Sciences.

Required Author Forms Disclosure forms provided by the authors are available with the online version of this article.

Conflict of interest The authors declare that they have no conflict of interest.

\section{References}

1. Kannian P, Green PL. Human T lymphotropic virus type 1 (HTLV1): molecular biology and oncogenesis. Viruses 2010;2:2037-2077.

2. Trevino A, Aguilera A, Caballero E, et al. Trends in the prevalence and distribution of HTLV-1 and HTLV-2 infections in Spain. Virol J 2012;9:71. 
3. Rafatpanah H, Hedayati-Moghaddam MR, Fathimoghadam F, et al. High prevalence of HTLV-1 infection in Mashhad, Northeast Iran: a population-based seroepidemiology survey. J Clin Virol 2011;52: 172-176.

4. Yamashiro T, Kamiya H, Miyara T, et al. CT scans of the chest in carriers of human T-cell lymphotropic virus type 1: presence of interstitial pneumonia. Acad Radiol 2012;19:952-957.

5. Gessain A, Cassar O. Epidemiological aspects and world distribution of HTLV-1 Infection. Front Microbiol 2012;3:388.

6. Azarpazhooh MR, Hasanpour K, Ghanbari M, et al. Human Tlymphotropic virus type 1 prevalence in northeastern Iran, Sabzevar: an epidemiologic-based study and phylogenetic analysis. AIDS Res Hum Retroviruses 2012;28:1095-1101.

7. Boostani R, Mellat Ardakani A, Ashrafi H. Khorasan disease: prevalence of HTLV-1 associated myelopathy/tropical spastic paraparesis (HAM/TSP) in west Azarbaijan from 2004 to 2007. Iran Red Crescent Medical J 2011;13:428-430.

8. Kalavi K, Moradi A, Tabarraei A. Population-based seroprevalence of HTLV-1 infection in Golestan Province, South East of Caspian Sea, Iran. Iran J Basic Med Sci 2013;16:225-228.

9. Ahmadi Ghezeldasht S, Shirdel A, Assarehzadegan MA, et al. Human T lymphotropic virus type I (HTLV-1) oncogenesis: molecular aspects of virus and host interactions in pathogenesis of adult $\mathrm{T}$ cell leukemia/ lymphoma (ATL). Iran J Basic Med Sci 2013;16:179-195.

10. Shoeibi A, Etemadi M, Moghaddam Ahmadi A, Amini M, Boostani R. "HTLV-1 Infection" twenty-year research in neurology department of Mashhad University of Medical Sciences. Iran J Basic Med Sci 2013;16:202-207.

11. Farid Hosseni R, Jabbari F, Shabestari M, et al. Human T lymphotropic virus type I (HTLV-1) is a risk factor for coronary artery disease. Iran J Basic Med Sci 2013;16:217-220.

12. Lepoutre V, Jain P, Quann K, Wigdahl B, Khan ZK. Role of resident CNS cell populations in HTLV-1-associated neuroinflammatory disease. Front Biosci 2009;14:1152-1168.

13. Kubota R, Hanada K, Furukawa Y, et al. Genetic stability of human T lymphotropic virus type I despite antiviral pressures by CTLs. J Immunol 2007;178:5966-5972.

14. Lomas M, Hanon E, Tanaka Y, Bangham CR, Gould KG. Presentation of a new $\mathrm{H}-2 \mathrm{D}(\mathrm{k})$-restricted epitope in the Tax protein of human T-lymphotropic virus type I is enhanced by the proteasome inhibitor lactacystin. J Gen Virol 2002;83:641-650.

15. Casseb J, Fukumori L, Vergara M, et al. Lack of tax diversity for tropical spastic paraparesis/human T-cell lymphotropic virus type 1 (HTLV-1) associated myelopathy development in HTLV-1-infected subjects in Sao Paulo, Brazil. Mem Inst Oswaldo Cruz 2006;101: 273-276.

16. Zhao T, Matsuoka M. HBZ and its roles in HTLV-1 oncogenesis. Front Microbiol 2012;3:247.

17. Matsuoka M, Green PL. The HBZ gene, a key player in HTLV-1 pathogenesis. Retrovirology 2009;6:71.

18. Zhao T, Yasunaga J, Satou Y, et al. Human T-cell leukemia virus type 1 bZIP factor selectively suppresses the classical pathway of NF-kappaB. Blood 2009;113:2755-2764.

19. Belrose G, Gross A, Olindo S, et al. Effects of valproate on Tax and HBZ expression in HTLV-1 and HAM/TSP T lymphocytes. Blood 2011;118:2483-2491.

20. Kchour G, Rezaee R, Farid R, et al. The combination of arsenic, interferon-alpha, and zidovudine restores an "immunocompetentlike" cytokine expression profile in patients with adult T-cell leukemia lymphoma. Retrovirology 2013;10:91.

21. Tattermusch S, Skinner JA, Chaussabel D, et al. Systems biology approaches reveal a specific interferon-inducible signature in HTLV-1 associated myelopathy. PLoS Pathog 2012;8:e1002480.

22. Hermine O, Wattel E, Gessain A, Bazarbachi A. Adult T cell leukaemia: a review of established and new treatments. BioDrugs 1998;10:447-462.
23. Carod-Artal FJ. [Immunopathogenesis and treatment of the myelopathy associated to the HTLV-1 virus]. Rev Neurol 2009;48:147155 (in Spanish).

24. Pot C, Chizzolini C, Vokatch N, et al. Combined antiviralimmunosuppressive treatment in human T-lymphotrophic virus 1Sjogren-associated myelopathy. Arch Neurol 2006;63:1318-1320.

25. Rafatpanah H, Rezaee A, Etemadi MM, et al. The impact of interferon-alpha treatment on clinical and immunovirological aspects of HTLV-1-associated myelopathy in northeast of Iran. J Neuroimmunol 2012;250:87-93.

26. Yamano Y, Sato T, Ando H, Araya N, Yagishita N. [The current and future approaches to the treatment of HTLV-1-associated myelopathy/tropical spastic paraparesis (HAM/TSP)]. Nihon Rinsho 2012;70:705-713

27. Oh U, Jacobson S. Treatment of HTLV-1-associated myelopathy/ tropical spastic paraparesis: toward rational targeted therapy. Neurol Clin 2008;26:781-797.

28. Izumo S, Goto I, Itoyama $\mathrm{Y}$, et al. Interferon-alpha is effective in HTLV-1-associated myelopathy: a multicenter, randomized, double-blind, controlled trial. Neurology 1996;46:1016-1021.

29. Yang YB, Zhang J, Leng ZP, Chen X, Song WQ. Evaluation of spasticity after stroke by using ultrasound to measure the muscle architecture parameters: a clinical study. Int J Clin Exp Med 2014; 7: 2712-2717.

30. Hajebrahimi S, Nourizadeh D, Hamedani R, Pezeshki MZ. Validity and reliability of the International Consultation on Incontinence Questionnaire-Urinary Incontinence Short Form and its correlation with urodynamic findings. Urol J 2012;9:685-690.

31. Seckiner I, Yesilli C, Mungan NA, Aykanat A, Akduman B. Correlations between the ICIQ-SF score and urodynamic findings. Neurourol Urodyn 2007;26:492-494.

32. Simsek A, Ozgor F, Yuksel B, et al. Female sexual function after transobturator tape in women with urodynamic stress urinary incontinence. SpringerPlus 2014;3:570.

33. Saifi B, Rezaee SA, Tajik N, et al. Th17 cells and related cytokines in unexplained recurrent spontaneous miscarriage at the implantation window. Reprod Biomed Online 2014;29:481-489.

34. Furtado Mdos S, Andrade RG, Romanelli LC, et al. Monitoring the HTLV-1 proviral load in the peripheral blood of asymptomatic carriers and patients with HTLV-associated myelopathy/tropical spastic paraparesis from a Brazilian cohort: ROC curve analysis to establish the threshold for risk disease. J Med Virol 2012;84:664-671.

35. Matsuzaki T, Nakagawa M, Nagai M, et al. HTLV-1 proviral load correlates with progression of motor disability in HAM/TSP: analysis of $239 \mathrm{HAM}$ /TSP patients including 64 patients followed up for 10 years. J Neurovirol 2001;7:228-234.

36. Vakili R, Sabet F, Aahmadi S, et al. Human T-lymphotropic virus type 1 (HTLV-1) proviral load and clinical features in Iranian HAM/ TSP patients: comparison of HTLV-1 proviral load in HAM/TSP patients. Iran J Basic Med Sci 2013;16:268-272.

37. Nakamura T, Shibayama K, Nagasato K, Matsuo H, Tsujihata M, Nagataki S. The efficacy of interferon-alpha treatment in human Tlymphotropic virus type-I-associated myelopathy. Jpn J Med 1990;29:362-367.

38. Feng J, Misu T, Fujihara K, et al. Th1/Th2 balance and HTLV-1 proviral load in HAM/TSP patients treated with interferon-alpha. $\mathrm{J}$ Neuroimmunol 2004;151:189-194.

39. Feng J, Misu T, Fujihara K, et al. Interferon-alpha significantly reduces cerebrospinal fluid CD4 cell subsets in HAM/TSP. J Neuroimmunol 2003;141:170-173.

40. Lezin A, Gillet N, Olindo S, et al. Histone deacetylase mediated transcriptional activation reduces proviral loads in HTLV-1 associated myelopathy/tropical spastic paraparesis patients. Blood 2007;110:3722-3728. 
41. Afonso PV, Mekaouche M, Mortreux F, et al. Highly active antiretroviral treatment against STLV-1 infection combining reverse transcriptase and HDAC inhibitors. Blood 2010;116:3802-3808.

42. Olindo S, Belrose G, Gillet N, et al. Safety of long-term treatment of HAM/TSP patients with valproic acid. Blood 2011;118:6306-6309.

43. Boostani R, Saber H, Etemadi M. Effects of danazol on clinical improvement of patients with human T-cell lymphotropic virus type I associated myelopathy/tropical spastic paraparesis (HAM/TSP): a placebo-controlled clinical trial. Iran J Basic Med Sci 2013;16:213-216.

44. Saito M, Bangham CR. Immunopathogenesis of human T-cell leukemia virus type-1-associated myelopathy/tropical spastic paraparesis: recent perspectives. Leukemia Res Treat 2012;2012:259045.
45. Gottlicher M. Valproic acid: an old drug newly discovered as inhibitor of histone deacetylases. Ann Hematol 2004;83(Suppl. 1):S91S92.

46. Bialer M, Yagen B. Valproic Acid: second generation. Neurotherapeutics 2007;4:130-137.

47. Holmes LB. Human teratogens: update 2010. Birth Defects Res A Clin Mol Teratol 2011;91:1-7.

48. Lloyd KA. A scientific review: mechanisms of valproate-mediated teratogenesis. Bioscience Horizons 2013;6:hzt003.

49. Kira J. Therapeutic benefits of an oral vitamin B1 derivative for human T lymphotropic virus type I-associated myelopathy/tropical spastic paraparesis (HAM/TSP). BMC Med 2013;11:183. 